\title{
Female Sexual Function and Perineal Pain Following Median versus Mediolateral Episiotomy in Primiparous Women: A Two-Year Follow up Study
}

\author{
Mohamed R*, Alaa Al-Halaby, Mohamed E and Wael G \\ Department of Obstetrics and Gynecology, Faculty of Medicine, Menoufia University, Egypt
}

Submission: December 09, 2017; Published: February 06, 2018

*Corresponding author: Mohamed Rezk, Department of Obstetrics and Gynecology, Faculty of Medicine, Menoufia University, Egypt, Tel: +201146256413, +201006237186; Email: m_rezk9207@yahoo.com

Keywords: primiparous women; Mediolateral episiotomy; Median episiotomy

Abbreviations: TFSFI: Arabic version of Female Sexual Function Index; VAS: Visual Analogue Scale; FSFI: Female Sexual Function Index; MLE:

Mediolateral; LE: Lateral

\section{Introduction}

Episiotomy is the most frequently performed surgical procedure for labour management especially when expedited delivery is warranted as in non-reassuring fetal heart rate tracing, a prolonged second stage of labour, or when operative vaginal delivery is indicated [1-3]. Postpartum perineal pain and dyspareunia affect about $42 \%$ of primiparous women within the first 2 weeks after vaginal delivery. Pain resolved in the majority of women within 2 months after delivery. Spontaneous second degree tears cause less perineal pain than episiotomies [4]. The aim of the study was to test the hypothesis whether the type of episiotomy (median versus mediolateral) can affect the degree of perineal pain and female sexual function over two years postdelivery or not.

\section{Materials and Methods}



Figure 1: Flow diagram of recruitment and retention of participants in the study. 


\section{Global Journal of Reproductive Medicine}

This prospective longitudinal study was conducted at the department of Obstetrics and Gynecology, Menoufia University hospital, Shibin El-Kom city, Menoufia governorate, Egypt during the period between September 2012 and September 2017. All procedures performed in the current study involving human participants were in accordance with the ethical standards of the Menoufia Faculty of Medicine research ethical committee and with the 1964 Helsinki declaration and its later amendments. Informed consent was obtained from all participants prior to commencing the study. Primarous women presented in active labour in the Emergency room were invited to participate. Standard management of labour according to the hospital policy was followed and the type of episiotomy performed was according to the Obstetrician's preference and experience. Women with perineal tears of any degree, those required operative vaginal delivery and those whose husband had sexual dysfunction or travelling abroad were excluded from the study. Out of 716 primiparous women who attended the delivery room, 496 women were enrolled after exclusion of non-responders and those with exclusion criteria. Patients were underwent either median episiotomy $(\mathrm{n}=242)$ or mediolateral episiotomy $(n=254)$. Sixty eight women dropped out and 428 completed the study as shown in Figure 1. Participants were divided into two groups according to the type of episiotomy as follows:

I. Group 1 (Median episiotomy group): Included 210 women to whom median episiotomy was done.

II. Group 2 (Mediolateral episiotomy group): Included 218 women to whom mediolateral episiotomy was done.

In either type of episiotomy, continuous suturing with absorbable chromic catgut number 0 was used for repair in all women. Participants were scheduled to attend the outpatient clinic at 6 weeks for method of contraception and follow up visits were scheduled at 3 months, 6 months, one year and two years following delivery. At each visit, women were assessed for the severity of perineal pain via using $10-\mathrm{cm}$ visual analogue scale, VAS (with anchor points of $0=$ no pain at all and $10=$ the most excruciating pain) and the numeric pain rating scale, The patient is asked to make three pain ratings, corresponding to current, best and worst pain experienced with the average of the 3 ratings was used to represent the patient's level of pain $(0=$ no pain, 1-3=mild pain, 4-6=moderate pain and $\geq 7=$ severe pain). Women were also asked to fill the validated Arabic version of Female Sexual Function Index (TFSFI). The scaling system of FSFI, which included questions on six topics included sexual desire, arousal, lubrication, orgasm, satisfaction, and pain. The lowest score was calculated as 2 and the highest score as 36. The total FSFI score under 26.5 was accepted as sexual dysfunction [5]. The severity of perineal pain and the changes of TFSFI scores over the two years following delivery were the main outcome measures.

\section{Statistical analysis}

Data was statistically analyzed by computer using SPSS version 22 (SPSS Inc, Chicago, IL, USA). Parametric data was expressed as means and standard deviations with student t-test and Mann Whitney test were used to compare between the two groups where appropriate, while non-parametric data was expressed as number and percent with Chi-squared test was used to compare categorical outcome. P value $\leq 0.05$ was considered to indicate significance and $\mathrm{p} \leq 0.001$ was considered to indicate strong significance.

\section{Results}

There was no significant difference between the two groups regarding the demographic data including age, duration of marriage, education level, income level, occupation, timing of postpartum contraception and method of contraception ( $p>0.05)$ as depicted in Table 1. There was no significant difference between the two groups regarding the severity of perineal pain using the visual analogue scale (VAS) and Female sexual function index (FSFI) individual components and total scores at 3 months, 6 months, one and two years after delivery $(p>0.05)$ as shown in Table 2. There was no significant difference between the two groups regarding the degree of perineal pain using the numeric pain rating scale at 3 months, 6 months, one and two years after delivery $(p>0.05)$ as revealed in Table 3 . There was no significant difference between the two groups regarding the number of women with female sexual dysfunction (FSFI score $<26.5$ ) at 3 months, 6 months, one and two years after delivery ( $p>0.05)$ as shown in Table 4. Figure 2 shows Female sexual dysfunction (FSFI score $<26.55$ ) among participants.

Table 1: Participants' characteristics.

\begin{tabular}{|c|c|c|c|c|}
\hline & Median group (n=210) & $\begin{array}{c}\text { Mediolateral group } \\
(\mathbf{n = 2 1 8})\end{array}$ & Student t-test & P-value \\
\hline Age (years) & $23.8 \pm 5.2$ & $24.1 \pm 4.9$ & 0.61 & $>0.05$ \\
\hline $\begin{array}{c}\text { Duration of marriage } \\
\text { (months) }\end{array}$ & $18.8 \pm 4.2$ & $19.2 \pm 3.9$ & 1.02 & $>0.05$ \\
\hline $\begin{array}{c}\text { Education level: -Primary } \\
\text {-Secondary } \\
\text {-Higher }\end{array}$ & 34 & 32 & 0.09 & $>0.05$ \\
\hline
\end{tabular}




\begin{tabular}{|c|c|c|c|c|}
\hline -Low & 50 & 54 & 0.01 & $>0.05$ \\
\hline -Moderate & 120 & 124 & 0.001 & $>0.05$ \\
\hline -High & 40 & 40 & 0.001 & $>0.05$ \\
\hline \multicolumn{5}{|c|}{ Occupation } \\
\hline -Employed & 120 & 128 & & \\
\hline -Unemployed & 90 & 90 & 0.00 & -0.00 \\
\hline \multicolumn{5}{|c|}{ Contraceptive Method } \\
\hline $\begin{array}{c}\text {-Fertility awareness } \\
\text { methods (\%) }\end{array}$ & 48 & 52 & 0.02 & $>0.05$ \\
\hline $\begin{array}{c}\text {-Intrauterine device (IUD) } \\
(\%)\end{array}$ & 122 & 120 & 0.29 & $>0.05$ \\
\hline $\begin{array}{c}\text {-Hormonal contraception } \\
(\%)\end{array}$ & 40 & 46 & 0.17 & $>0.05$ \\
\hline \multicolumn{5}{|c|}{ Timing of Contraceptive Use } \\
\hline -Before 6 months & 80 & 82 & 0.01 & $>0.05$ \\
\hline -At 6-12 months & 62 & 72 & 0.68 & $>0.05$ \\
\hline -After 12 months & 20 & 12 & 1.89 & $>0.05$ \\
\hline
\end{tabular}

Table 2: Perineal pain and Female sexual function index (FSFI) among participants.

\begin{tabular}{|c|c|c|c|c|}
\hline & Median Group $(n=210)$ & $\begin{array}{l}\text { Mediolateral Group } \\
\qquad(n=218)\end{array}$ & Student t-test & P-value \\
\hline \multicolumn{5}{|c|}{ Severity of perineal pain [VAS] } \\
\hline -At 3 months & $6.4 \pm 2.3$ & $6.1 \pm 2.4$ & 1.32 & $>0.05$ \\
\hline -At 6 months & $5.1 \pm 1.8$ & $5.2 \pm 1.6$ & 0.61 & $>0.05$ \\
\hline -At one year & $3.2 \pm 1.1$ & $3.1 \pm 1.2$ & 0.9 & $>0.05$ \\
\hline -At two years & $2.9 \pm 1.6$ & $2.8 \pm 1.4$ & 0.69 & $>0.05$ \\
\hline \multicolumn{5}{|c|}{ FSFI at 3 months } \\
\hline -Desire & $3.2 \pm 1.1$ & $3.1 \pm 1.2$ & 0.9 & $>0.05$ \\
\hline -Arousal & $3.6 \pm 1.4$ & $3.5 \pm 1.5$ & 0.71 & $>0.05$ \\
\hline -Lubrication & $3.8 \pm 1.2$ & $3.7 \pm 1.3$ & 0.83 & $>0.05$ \\
\hline -Orgasm & $3.7 \pm 1.5$ & $3.8 \pm 1.4$ & 0.71 & $>0.05$ \\
\hline -Satisfaction & $4.4 \pm 1.6$ & $4.5 \pm 1.5$ & 0.67 & $>0.05$ \\
\hline -Pain & $4.6 \pm 1.7$ & $4.5 \pm 1.6$ & 0.63 & $>0.05$ \\
\hline Total & $22.4 \pm 6.4$ & $22.6 \pm 6.1$ & 0.33 & $>0.05$ \\
\hline \multicolumn{5}{|c|}{ FSFI at 6 months } \\
\hline -Desire & $3.6 \pm 1.5$ & $3.7 \pm 1.4$ & 0.71 & $>0.05$ \\
\hline -Arousal & $3.8 \pm 1.7$ & $3.9 \pm 1.6$ & 0.63 & $>0.05$ \\
\hline -Lubrication & $3.9 \pm 1.5$ & $3.8 \pm 1.6$ & 0.67 & $>0.05$ \\
\hline -Orgasm & $3.8 \pm 1.6$ & $3.7 \pm 1.5$ & 0.67 & $>0.05$ \\
\hline -Satisfaction & $4.6 \pm 1.8$ & $4.5 \pm 1.7$ & 0.59 & $>0.05$ \\
\hline -Pain & $4.4 \pm 1.4$ & $4.3 \pm 1.5$ & 0.71 & $>0.05$ \\
\hline Total & $24.6 \pm 8.4$ & $24.5 \pm 8.2$ & 0.12 & $>0.05$ \\
\hline \multicolumn{5}{|c|}{ FSFI at one Year } \\
\hline -Desire & $3.8 \pm 1.6$ & $3.7 \pm 1.7$ & 0.63 & $>0.05$ \\
\hline -Arousal & $3.9 \pm 1.8$ & $3.8 \pm 1.9$ & 0.56 & $>0.05$ \\
\hline -Lubrication & $3.9 \pm 1.8$ & $3.8 \pm 1.8$ & 0.57 & $>0.05$ \\
\hline
\end{tabular}


Global Journal of Reproductive Medicine

\begin{tabular}{|c|c|c|c|c|}
\hline & & & & \\
\hline -Orgasm & $3.9 \pm 1.7$ & $3.8 \pm 1.6$ & 0.63 & $>0.05$ \\
\hline -Satisfaction & $4.8 \pm 1.9$ & $4.9 \pm 1.7$ & 0.57 & $>0.05$ \\
\hline -Pain & $4.2 \pm 1.2$ & $4.1 \pm 1.1$ & 0.9 & $>0.05$ \\
\hline Total & $26.4 \pm 7.4$ & $26.5 \pm 7.5$ & 0.14 & $>0.05$ \\
\hline \multicolumn{5}{|c|}{ FSFI at two years } \\
\hline -Desire & $3.9 \pm 1.8$ & $3.8 \pm 1.8$ & 0.57 & $>0.05$ \\
\hline -Arousal & $3.9 \pm 1.9$ & $3.8 \pm 1.8$ & 0.56 & $>0.05$ \\
\hline -Lubrication & $3.8 \pm 1.8$ & $3.7 \pm 1.7$ & 0.59 & $>0.05$ \\
\hline -Orgasm & $3.7 \pm 1.9$ & $3.8 \pm 1.7$ & 0.57 & $>0.05$ \\
\hline -Satisfaction & $4.8 \pm 1.9$ & $4.9 \pm 1.8$ & 0.56 & $>0.05$ \\
\hline -Pain & $3.9 \pm 1.1$ & $3.8 \pm 1.1$ & 0.94 & $>0.05$ \\
\hline Total & $26.6 \pm 4.2$ & $26.7 \pm 4.1$ & 0.25 & $>0.05$ \\
\hline
\end{tabular}

Table 3: Degree of perineal pain using the numeric pain rating scale among participants.

\begin{tabular}{|c|c|c|c|c|}
\hline & Median Group $(n=210)$ & $\begin{array}{c}\text { Mediolateral Group } \\
(\mathrm{n}=218)\end{array}$ & Chi square Test & P-value \\
\hline \multicolumn{5}{|c|}{ Perineal Pain at 3 months } \\
\hline -Mild & $98(46.6 \%)$ & $108(49.5 \%)$ & 0.25 & $>0.05$ \\
\hline -Moderate & $76(36.2 \%)$ & $74(33.9 \%)$ & 0.15 & $>0.05$ \\
\hline -Severe & $36(17.2 \%)$ & $36(16.6 \%)$ & 0.001 & $>0.05$ \\
\hline \multicolumn{5}{|c|}{ Perineal Pain at 6 months } \\
\hline -Mild & $122(58.1 \%)$ & $130(59.6 \%)$ & 0.05 & $>0.05$ \\
\hline -Moderate & $62(29.5 \%)$ & $60(27.5 \%)$ & 0.12 & $>0.05$ \\
\hline -Severe & $26(12.4 \%)$ & $28(12.9 \%)$ & 0.001 & $>0.05$ \\
\hline \multicolumn{5}{|c|}{ Perineal Pain at one year } \\
\hline -Mild & $138(65.7 \%)$ & $144(66.1 \%)$ & 0.001 & $>0.05$ \\
\hline -Moderate & $50(23.8 \%)$ & $52(23.8 \%)$ & 0.01 & $>0.05$ \\
\hline -Severe & $22(10.5 \%)$ & $22(10.1 \%)$ & 0.001 & $>0.05$ \\
\hline \multicolumn{5}{|c|}{ Perineal Pain at two years } \\
\hline -Mild & $148(70.5 \%)$ & $160(73.4 \%)$ & 0.32 & $>0.05$ \\
\hline -Moderate & $50(23.8 \%)$ & $48(22.1 \%)$ & 0.11 & $>0.05$ \\
\hline -Severe & $12(5.7 \%)$ & $10(4.5 \%)$ & 0.1 & $>0.05$ \\
\hline
\end{tabular}

Table 4: Female sexual dysfunction (FSFI score < 26.5) among participants.

\begin{tabular}{|c|c|c|c|c|}
\hline & Median Group (n=210) & $\begin{array}{c}\text { Mediolateral Group } \\
(\mathbf{n = 2 1 8})\end{array}$ & Chi square Test & P-value \\
\hline $\begin{array}{c}\text { Female sexual dysfunction } \\
\text { at 3 months }\end{array}$ & $146(69.5 \%)$ & $134(61.5 \%)$ & 2.72 & $>0.05$ \\
\hline $\begin{array}{c}\text { Female sexual dysfunction } \\
\text { at 6 months }\end{array}$ & $112(53.3 \%)$ & $102(46.8 \%)$ & 1.1 & $>0.05$ \\
\hline $\begin{array}{c}\text { Female sexual dysfunction } \\
\text { at one year }\end{array}$ & $86(40.9 \%)$ & $78(35.8 \%)$ & 0.1 & $>0.05$ \\
\hline $\begin{array}{c}\text { Female sexual dysfunction } \\
\text { at two years }\end{array}$ & $42(20 \%)$ & $40(18.3 \%)$ & $>0.05$ \\
\hline
\end{tabular}




\section{Global Journal of Reproductive Medicine}

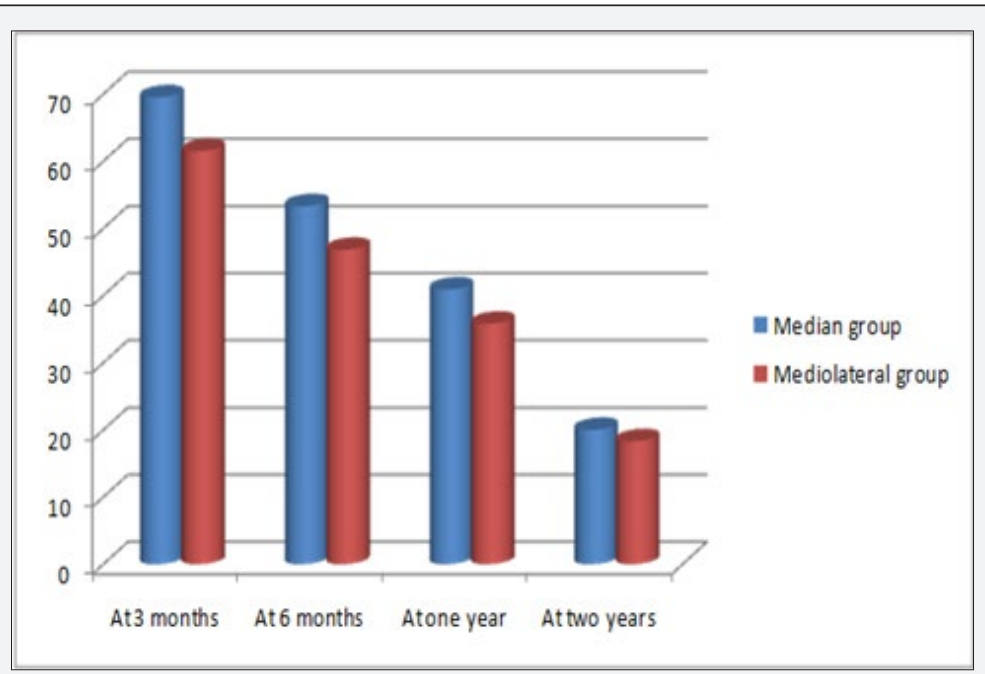

Figure 2 : Female sexual dysfunction (FSFI score $<26.55$ ) among participants.

\section{Discussion}

Moderate to severe perineal pain affects $53.4 \%, 41.9 \%$, $34.3 \%$ and $29.5 \%$ of primaparous women who underwent median episiotomy at 3 months, 6 months, one and two years following delivery compared to $50.5 \%, 40.4 \%, 33.9 \%$ and $26.6 \%$ of primaparous women underwent mediolateral episiotomy respectively in the current study. Perineal trauma was more common among primiparous women with the incidence of perineal pain among women to whom episiotomy was performed $97 \%$ at day one and $71 \%$ at day 7 which decreased at 6 weeks postpartum [6]. In a previous randomized trial, morbidity including perineal damage by tears, pain at perineum and dyspareunia, was greater among 100 patients who were given a mediolateral episiotomy compared to 100 patients delivered without episiotomy when assessed after the puerperium [7]. Limiting perineal trauma during delivery is important for the resumption of sexual intercourse after delivery. Routine episiotomy should be avoided to minimize perineal trauma [8].

In this study, the rates of female sexual dysfunction were $69.5 \%, 53.3 \%, 40.9 \%$ and $20 \%$ among primaparous women who underwent median episiotomy at 3 months, 6 months, one and two years following delivery compared to $61.5 \%, 46.8 \%$, $35.8 \%$ and $18.3 \%$ among primaparous women underwent mediolateral episiotomy respectively. High degree perineal tears (third and forth) negatively affect female sexual function up to one year after delivery [9]. A recent prospective study evaluated dyspareunia and sexual function at 3-12 months after vaginal delivery among 190 Thai primiparous women with episiotomy. Sexual dysfunction $(\mathrm{FSFI}<26.5)$ was demonstrated in $66.7 \%$ at 3 months, $31.0 \%$ at 6 months, and $14.9 \%$ at 12 months. From 3 to 12 months, the median total FSFI scores in all domains increased significantly [10].

Another recent prospective comparative study evaluating peripartum outcome of a vaginal delivery after mediolateral (MLE) or lateral (LE) episiotomy with 306 MLE and 342 LE, revealed $98.0 \%$ of women after MLE and $97.7 \%$ after LE resumed sexual intercourse within 6 months after delivery with $15.6 \%$ of women after MLE and $16.1 \%$ after LE suffered from considerable dyspareunia [11]. Any increase in the extent of perineal trauma is associated with the highest scores in the self-assessment of perceived perineal pain and long term persistent symptoms. Furthermore, the presence of an episiotomy is a conditioning factor for the sexual function after delivery [12]. Repair of episiotomy in the current study was by continuous suturing with absorbable suture, based on a previous Cochrane review which concluded that the continuous suturing techniques for perineal closure, compared with interrupted methods, are associated with less short-term pain, need for analgesia and suture removal [13]. The high response rate and the longer follow up periods constitute the main strength of the current study. Inability to trace the impact of episiotomy on anal and urinary functions constitutes unintended limitation of this study.

\section{Conclusion}

Median and mediolateral episiotomy have comparable degrees of perineal pain and female sexual dysfunction which is worst earlier after delivery and improves over time.

\section{References}

1. Klein MC, Gauthier RJ, Robbins JM, Kaczorowski J, Jorgensen SH, et al. (1994) Relationship of episiotomy to perineal trauma and morbidity, sexual dysfunction, and pelvic floor relaxation. Am J Obs Gyne 171(3): 591-598.

2. Hartmann K, Viswanathan M, Palmieri R, Gartlehner G, Thorp J, et al. (2005) Outcomes of routine episiotomy: a systematic review. JAMA 293(17): 2141-2148.

3. Carroli C, Belizan J (2008) Episiotomy for vaginal birth. Cochrane Library. Cochrane Database Syst Rev 1: CD000081.

4. Andrews V, Thakar R, Sultan AH, Jones PW (2008) Evaluation of postpartum perineal pain and dyspareunia -a prospective study. Eur J Obstet Gynecol Reprod Biol 137(2):152-156.

5. Wiegel M, Meston C, Rosen R (2005) The Female Sexual Function Index (FSFI): Cross-validation and Development of Clinical Cutoff Scores. J Sex Marital Ther 31(1): 1-20. 
6. Macarthur AJ, Macarthur C (2004) Incidence, severity, and determinants of perineal pain after vaginal delivery: a prospective cohort study. Am Obstet Gynecol 191(4): 1199-11204.

7. Islam A, Hanif A, Ehsan A, Arif S, Niazi SK, et al. (2013) Morbidity from episiotomy. J Pak Med Assoc. 63(6): 696-701.

8. Rathfisch G, Dikencik BK, Kizilkaya Beji N, Comert N, Tekirdag AI, et al. (2010) Effects of perineal trauma on postpartum sexual function. J Adv Nurs 66(12): 2640-2649.

9. Sayed Ahmed WA, Kishk EA, Farhan RI, Khamees RE (2017) Female sexual function following different degrees of perineal tears. Int Urogynecol J 28(6): 917-921.

10. Chayachinda C, Titapant V, Ungkanungdecha A (2015) Dyspareunia and sexual dysfunction after vaginal delivery in Thai primiparous women with episiotomy. J Sex Med 12(5): 1275-1282.
11. Necesalova P, Karbanova J, Rusavy Z, Pastor Z, Jansova M, et al. (201M) ediolateral versus lateral episiotomy and their effect on postpartum coital activity and dyspareunia rate 3 and 6 months postpartum. Sex Reprod Healthc 8: 25-30.

12. Persico G, Vergani P, Cestaro C, Grandolfo M, Nespoli A (2013) Assessment of postpartum perineal pain after vaginal delivery: prevalence, severity and determinants. A prospective observational study. Minerva Ginecol 65(6): 669-678.

13. Kettle C, Dowswell T, Ismail KM (2012) Continuous and interrupted suturing techniques for repair of episiotomy or second-degree tears. Cochrane Database Syst Rev 11: CD000947.

\section{Your next submission with Juniper Publishers will reach you the below assets}

- Quality Editorial service

- Swift Peer Review

- Reprints availability

- E-prints Service

- Manuscript Podcast for convenient understanding

- Global attainment for your research

- Manuscript accessibility in different formats

( Pdf, E-pub, Full Text, Audio)

- Unceasing customer service

Track the below URL for one-step submission https://juniperpublishers.com/online-submission.php 